\title{
SINGULAR EVOLUTION PROBLEMS, REGULARIZATION, AND APPLICATIONS TO PHYSICS, ENGINEERING, AND BIOLOGY
}

\author{
GÜNTER LUMER \\ Institute of Mathematics and Informatics, University of Mons-Hainaut \\ Avenue Maistriau, 15, 7000 Mons, Belgium \\ E-mail:dufour@umh.ac.be
}

0. Introduction. We consider general (linear inhomogeneous) evolution problems of the type

$$
u^{\prime}=A u+F(t), u(0)=f,
$$

on $[0, a[, a>0$, in a Banach space $X, A$ being any closed linear operator in $X$ (i.e. with domain $D(A)$ and range $I(A)$ in $X)\left({ }^{1}\right), F \in L_{\text {loc }}^{1}([0, a[, X)$. A problem of this kind may be regular (i.e. have a unique $C^{1}$ solution on $[0, a[$ for every initial value $f \in X$ ), however this is often not the case. Here we shall direct our attention to the matter of how to deal with singular problems (specifically problems not regular at $t=0)\left({ }^{2}\right)$, and describe briefly some of the methods and results developed recently by many people including the author, to treat and often solve in a useful/usable sense the mentioned singular problems $\left({ }^{3}\right)$.

Let us start by recalling that much interesting work in the just mentioned direction was done in the last ten years or so (and in part even earlier), first by using ( $n$-times) integrated semigroups (or $C$-regularized semigroups) in lieu of the usual semigroups to

1991 Mathematics Subject Classification: 34-02, 34G10, 34G99, 47-02, 47D06, 35-02, 35A35, 35A25, 35D05, 35Q99.

The author's research mentioned in the present paper was supported in part by the European Union (EU) project "Evolutionary Systems", contract SC1*CT90-0464.

The paper is in final form and no version of it will be published elsewhere.

$\left({ }^{1}\right)$ The set of all such operators (closed or not) will be denoted $\mathcal{L}(X)$.

$\left({ }^{2}\right)$ Regular problems appear as special cases in this approach.

$\left({ }^{3}\right)$ Of course the present brief article has no pretense of being even approximately a complete survey of results or references; however, we believe it provides enough information on recent progress (results, methods, applications) and trends in the area mentioned, to be of interest to both people working on such and related matters, and people wanting to learn about them. 
treat singular problems. Arendt [1], Da Prato [12], Kellermann and Hieber [17], Neubrander [33], deLaubenfels [21], Lumer [23], Miyadera [32], are some references (among many others) on this work $\left({ }^{4}\right)$.

We now take up directly the description of the recently developed regularization approach based on the systematic use of local generalized solutions and generalized evolution operators. This approach widely extends $\left({ }^{5}\right)$ the earlier ones mentioned in the above paragraph; first developed by the author since 1990 at the level of local integrated solutions [23], [24], [26], [30], and then at the very general level of local $K(t)$-generalized solutions and corresponding evolution operators $\mathcal{S}_{K}(t)$ by I. Cioranescu and the author in 1994, [8], [9] (in particular for " $K$-convoluted semigroups" in [9]). This goes as follows:

1. $K(t)$-regularized solutions and evolution operators. Keeping the notations of Section 0, let $K:[0, a[\rightarrow \mathcal{B}(X)$ (the space of all bounded linear operators on $X$, i.e. with $D(\cdot)=X, I(\cdot) \subset X)$ be strongly continuous, here for simplicity even strongly $C^{1}$, and satisfying $K(t) K(s)=K(s) K(t), K(t) A=A K(t)$ on $D(A)$, for $0 \leq s, t<a$. We also assume that $A$ has the uniqueness property $\left({ }^{6}\right)$.

We write $K(0)=C, K(t)=C+K_{0}(t)$; we call $K$ a kernel.

The original problem (1) is now replaced by the regularized problem:

$$
v^{\prime}=A v+K(t) f+F_{K}(t), \quad v(0)=0,
$$

where $v^{\prime}=v_{K}^{\prime}$ stands for $v^{\prime}(t)=v^{\prime}(t, f)=v_{K}^{\prime}(t, f)$,

$$
F_{K}(t)=(K \star F)(t)=\int_{0}^{t} K(t-s) F(s) d s,
$$

and $v$ is a $C^{1}$ solution of (2) on [0,a[. $v_{K}$ is called a $K$-strong generalized solution of (1) ( $K$-s.g.s.) starting at $f$, and $v_{K}^{\prime}$ is called a $K$-mild generalized solution of (1) ( $K$-m.g.s.) starting at $f$.

Nothing is presumed about the set of initial values $f$ for which $(2)$ can be solved. We define:

$$
\begin{array}{r}
Z_{K}=Z_{K}(a)=\left\{f \in X: \text { there exists a } K \text {-s.g.s. } v_{K}(t, f) \text { on }[0, a[\text { of }(1)\right. \\
\text { with } F=0 \text { starting at } f\},
\end{array}
$$

and define the $K(t)$-evolution operators $\mathcal{S}_{K}(t)$ via

$$
\mathcal{S}_{K}(t) f=v_{K}^{\prime}(t, f), \quad 0 \leq t<a, \text { for } f \in Z_{K}(a) .
$$

When $K(t)=$ constant $=C$, we write simply $Z_{C}, \mathcal{S}_{C}(t)$; when $K(t)$ is "scalar", i.e. a scalar function multiplying the identity operator 1 , we omit 1 and identify $K(t)$ with the scalar part.

In the present general context the operators $\mathcal{S}_{K}(t)$ satisfy specific functional equations, depending on $K(\cdot)$ of course, which we describe below. We cannot give the proof for the

\footnotetext{
$\left({ }^{4}\right)$ More details and examples of the latter will appear below.

$\left({ }^{5}\right)$ As will be seen in a moment.

$\left({ }^{6}\right)$ I.e. $u=0$ on any $\left[0, a\left[\right.\right.$ on which $u^{\prime}=A u$ with $u(0)=0$ is satisfied.
} 
general case here, but it will be instructive to prove a very easy particular case, which we do next.

Proposition 1. Suppose $K(\cdot)=$ constant $=C \in \mathcal{B}(X)$. Let $0 \leq t<a, 0 \leq s$, $t+s<a, f \in Z_{C}(a)$. Then $\mathcal{S}_{C}(t) f \in Z_{C}(a-t)$, and

$$
C \mathcal{S}_{C}(t+s) f=\mathcal{S}_{C}(s) \mathcal{S}_{C}(t) f
$$

Remark 2. We can state also: $C \mathcal{S}_{C}(t+s)=\mathcal{S}_{C}(s) \mathcal{S}_{C}(t)=\mathcal{S}_{C}(t) \mathcal{S}_{C}(s)$ on $Z_{K}(a)$ for $0 \leq s, t, t+s<a, \mathcal{S}_{C}(t): Z_{C}(a) \rightarrow Z_{C}(a-t)$. (It is the functional equation satisfied by the " $C$-semigroups" mentioned in our introduction.)

Proof of Proposition 1. Since $f \in Z_{C}(a)$, we can consider $v(\cdot)=v_{C}(\cdot, f)$. For $0 \leq s<a-t$ set $w(s)=C(v(t+s)-v(t))$. Then $w(0)=0, \exists w^{\prime}(s)=C v^{\prime}(t+s)=$ $C(A v(t+s)+C f)=A C(v(t+s)-v(t))+C(A v(t)+C f)=A w(s)+C \mathcal{S}_{C}(t) f$, using $(3)$, $(2),(4)$. This proves that $\mathcal{S}_{C}(t) f \in Z_{C}(a-t)$ with $\mathcal{S}_{C}(s) \mathcal{S}_{C}(t) f=w^{\prime}(s)=C v^{\prime}(t+s)=$ $C v_{C}^{\prime}(t+s, f)=C \mathcal{S}_{C}(t+s) f$.

Of course in general no such simple arguments as above will suffice. Among the analytic tools most useful, and used throughout the whole theory, is the following extremely general variant of the so-called "variation of parameters formula", Duhamel formula, stated next.

TheOREM 3. Consider any $K$ as defined above, $f \in Z_{K}=Z_{K}(a), F:\left[0, a\left[\rightarrow Z_{K}\right.\right.$ and $\mathcal{S}_{K}(\cdot) F(\cdot):[0, a[\times[0, a[\rightarrow X$ strongly continuous. Then

$$
w(t)=\mathcal{S}_{K}(t) f+\int_{0}^{t} \mathcal{S}_{K}(t-s) F(s) d s,
$$

$0 \leq t<a$, is a K-m.g.s. of (1) starting at $f$.

Using this and other facts one can prove general functional equations. In the scalar case one has:

THEOREM 4. Let $K$ be any scalar kernel with $K(0)=0,0 \leq s, t<a, t+s<a$. Then $Z_{K}(t): Z_{K}(a) \rightarrow Z_{K}(a-t)$, and

$$
\begin{aligned}
\mathcal{S}_{K}(s) \mathcal{S}_{K}(t)= & \int_{0}^{t+s} K^{\prime}(t+s-r) \mathcal{S}_{K}(r) d r-\int_{0}^{t} K^{\prime}(t+s-r) \mathcal{S}_{K}(r) d r \\
& -\int_{0}^{s} K^{\prime}(t+s-r) \mathcal{S}_{K}(r) d r, \quad \text { on } Z_{K}(a) .
\end{aligned}
$$

One has a (more complicated) general formula in the non-scalar case.

An important particular case occurs for $K(t)=K_{n}(t)=t^{n} / n !, n=1,2,3, \ldots$ In that situation (where we write $\mathcal{S}_{n}(t)$ for $\mathcal{S}_{K}(t), Z_{n+1}$ for $Z_{K_{n}}$, and $S_{n}(t)$ when in addition $Z_{n+1}=X$, see [23], [30]), (7) becomes (on $\left.Z_{n+1}(a)\right)$

$$
\mathcal{S}_{n}(s) \mathcal{S}_{n}(t)=\int_{t}^{t+s} \frac{(t+s-r)^{n-1}}{(n-1) !} \mathcal{S}_{n}(r) d r-\int_{0}^{s} \frac{(t+s-r)^{n-1}}{(n-1) !} \mathcal{S}_{n}(r) d r
$$


a functional equation which serves as basis for the definition of $n$-times integrated semigroups ( $n$-i.s.g.) in [1]. More precisely, the latter are defined in [1] as a family $\left\{S_{n}(t) \in \mathcal{B}(X)\right\}_{t>0}$ with $t \mapsto S_{n}(t)$ strongly continuous, $S(0)=0$, satisfying (8) for $0 \leq s, t$. (We always implicitly assume our $n$-i.s.g. are non-degenerate, i.e. $S_{n}(t) f=0$ $\forall t>0$ implies $f=0,[1],[23]$.) One has (see [23], [30])

THEOREM 5. In the above context, $\left\{\mathcal{S}_{n}(t)\right\}_{t \geq 0}$ is an n-times integrated semigroup iff $Z_{n+1}=X$. On the other hand given an n-times integrated semigroup (as defined above following [1]) $\left\{S_{n}(t)\right\}_{t \geq 0}$, there is a uniquely determined $A$ in $X$ (the "generator" of $\left.S_{n}(t)\right)$ such that $S_{n}(t)=\mathcal{S}_{n}(t, A)$, i.e. $\mathcal{S}_{n}(t)$ with respect to $A$ for every $t \geq 0$.

So we see exactly how $n$-i.s.g. are special cases of $K(t)$-evolution operators; similarly for local $n$-i.s.g. [2], see [30]; also a similar situation holds for $C$-semigroups.

Before looking at the use of "higher order kernels", i.e. kernels more regularizing than $t^{n} / n$ ! (or $C$ ), we shall in the following two sections give some details, and some of the numerous useful applications, concerning integrated semigroups (i.s.g.).

2. 2-times integrated solutions, locally lipschitz i.s.g., and irregular analytic semigroups. Applications. A number of important applied problems (in physics, engineering, biology) give rise to singular problems which become however well-posed (on $X)$ in terms of $K$-s.g.s. with $K(t)=K_{n}(t), n=1$, i.e. in terms of generalized solutions which are "2-times integrated solutions" $\left({ }^{7}\right)$, the mild solutions (1-m.g.s.) being then given by the i.s.g. $S_{K_{n}}(t)=S_{1}(t)(n=1)$, which we denote simply $S(t)$. (See Theorem 5 and the paragraph preceding it.)

Since often the applied problems mentioned are formulated in terms of second order parabolic PDEs (and in sup-norm) the resulting i.s.g. have additional properties: (i) the i.s.g. $S(t)$ are locally lipschitz (loc. lip.) which means by definition that $\forall \delta>0$ $\exists M(\delta)>0$ such that $\|S(t)-S(t)\| \leq M(\delta)|t-s| \forall 0 \leq s, t \leq \delta\left(^{8}\right)$; (ii) $S(t)$ is an analytic loc.lip. i.s.g. (extending analytically to $S(z)$ in some angular neighborhood of the positive half axis $\{t>0\}$; for the exact definition see [27], or [30]). Moreover the latter $S(z)$ are very closely related to the "irregular bounded analytic semigroups" defined like the usual bounded analytic semigroups except that no continuity as $z \rightarrow 0$ is assumed (see [27]). The following result plays in these matters an essential role ([27], [30]).

THEOREM 6. $S(z)$ is a lip. analytic i.s.g. of angle $\alpha, 0<\alpha \leq \pi / 2$ (with angular domain $\left.\Gamma_{\alpha}\right)$ iff $S^{\prime}(z)=Q(z)$ is a irregular bounded analytic semigroup of angle $\alpha . A \in \mathcal{L}(X)$ is the generator of such a $S(z), Q(z)=S^{\prime}(z)$, (both having automatically the same generator,) iff $\exists M>0,0<\alpha \leq \pi / 2$ such that $\exists R(z, A)$ for $z \in \Gamma_{\alpha+\pi / 2}$ and for such $z\left({ }^{9}\right)$,

$$
\|R(z, A)\| \leq M /|z| \text {. }
$$

See [27], [30], [13], [35]. Actually the theory of i.s.g. is quite useful in establishing the just mentioned results, and in related investigations.

\footnotetext{
$\left({ }^{7}\right)$ Recall that convolution by $K_{n}(\cdot)$ is $(n+1)$-times iterated integration.

$\left({ }^{8}\right)$ "lip" instead of "loc.lip." is defined similarly (see [30], [27]).

$\left({ }^{9}\right)$ We use below the standard notation for the resolvent of $A, R(z, A)=(z-A)^{-1} \in \mathcal{B}(X)$.
} 
For general loc.lip. i.s.g. (without analyticity being necessarily present) one has the following essential result and formula ([23], [30], see also [37]).

TheOrem 7. $A \in \mathcal{L}(X)$ is the generator of a loc.lip. i.s.g. $S(t)$ iff $\exists M, \omega \geq 0$ such that $R(\lambda, A)$ exists $\forall \lambda>\omega$, and $\left\|(R(\lambda, A))^{n}\right\| \leq M /(\lambda-\omega)^{n}, n=1,2,3 \ldots$ If in addition $\exists A^{-1} \in \mathcal{B}(X)$ (which under the circumstances is not a major assumption) then $S(t)$ has the representation

$$
S(t)=\left(e^{t A_{0}}-1\right) A^{-1}
$$

where $A_{0}$ is the "part of $A$ in $\overline{D(A)}$ " (i.e. the largest restriction of $A$ operating in $\overline{D(A)}$ ).

If $A(x, D)$ is a second order strongly elliptic operator with real $C^{\infty}$ coefficients defined on the closure of a bounded domain $\Omega \subset \mathbb{R}^{N}$ with smooth boundary $\left.{ }^{10}\right), X=C(\bar{\Omega})$, then operator $A$ in $\mathcal{L}(X)=\mathcal{L}(C(\bar{\Omega}))$ defined by

$$
\begin{aligned}
D(A) & =\left\{f \in W^{2, p}(\Omega) \cap C_{0}(\Omega): A(x, D) f \in C(\bar{\Omega})\right\}, \\
A f & =A(x, D) f, \quad p>N,
\end{aligned}
$$

is independent of $p$ and, while not densely defined, satisfies the assumptions of Theorem 6 (and also of Theorem 7).

This permits the use of the above methods and results in many applied singular problems, as mentioned earlier, expressed by initial value-boundary value PDE problems of the type

$$
\begin{aligned}
& \frac{\partial u}{\partial t}=A(x, D) u+F(t, x) \quad \text { in }[0, a[\times \bar{\Omega}, \\
& u(0, x)=f(x), \quad x \in \bar{\Omega}, \\
& B u(t, \xi)=\varphi(t, \xi) \quad \text { in }] 0, a[\times \partial \Omega,
\end{aligned}
$$

$B$ a boundary operator (in what follows of trace type). In the corresponding Banach space formulation in which we then apply the above methods and results, we write

$$
\begin{aligned}
& u^{\prime}=\widehat{A} u+F(t), \\
& u(0)=f, \\
& B u(t)=\varphi(t) \in H, \quad t>0 .
\end{aligned}
$$

Everything is written in a single space $X$, by identifying the boundary values with their $\widehat{A}$-harmonic (i.e. $A(x, D)$-harmonic) extensions to $\bar{\Omega}$ in the classical situation, and letting in the general Banach space situation $B: X \rightarrow H$ take values in the subspace $H$ of $X$ (thought of as the abstract replacement of the space of $\widehat{A}$-harmonic functions). The operator $\widehat{A}$ appearing in (13) is closely related to the operator $A$ defined in (11) for the classical situation $X=C(\bar{\Omega})$, but not equal in general (indeed for $u \in D(\hat{A})$ satisfying (12) - in the classical case - $B u(t)=\varphi(t)$ in general not 0 , hence $u(t) \notin D(A)$ in general), and it is easily seen that $u(t)=w(t)+\varphi(t)$ with $w(t) \in D(A)$. In the general Banach space setup in which we consider (13), the classical properties of the $A$ of (11) as described

$\left({ }^{10}\right)$ We make strong regularity assumptions for simplicity, actually much less smoothness is necessary. 
above are replaced by the corresponding general simple assumptions:

(14) $A$ generates an irregular bounded analytic s.g. on $X, Q(t)$, and $\exists A^{-1} \in \mathcal{B}(X)$, $\widehat{A}$ being then derived from $A$ via

$$
\begin{aligned}
& D(\widehat{A})=D(A) \oplus H, \quad(D(A) \cap H=0), \\
& \widehat{A}(f+g)=A f \quad(\text { for } f \in D(A), g \in H) .
\end{aligned}
$$

In this context, we deal in the next section with a large class of singular parabolic problems involving variable boundary values, abrupt changes in boundary conditions, and highly singular interface transitions. However, before we take up these matters, of extremely recent vintage, in the next section, let us say that there are of course many other applications of i.s.g. and/or related irregular analytic semigroups (applications, and problems to which these methods can be applied), such as: the parabolic boundary value problems treated by Sinestrari and von Wahl in [36], as well as non-parabolic problems of considerable interest in mathematical biology (renewal equations and related equations of population dynamics) as in [24] and references given there to similar/related ample work by Ph. Clément, O. Diekmann and coworkers, see also [11] and references given there.

3. Solutions for problems of parabolic type with variable boundary values. Heat shocks. Singular transition problems. We take up (13), under (14) and (15). The change of variable $u(t)=w(t)+\varphi(t)$ in (13) gives $B w(t)=0, w(t) \in D(A)$.

$$
\begin{aligned}
& w^{\prime}=A w-\varphi^{\prime}(t)+F(t), \\
& w(0)=f-\varphi(0) .
\end{aligned}
$$

Now (16) can be solved using results of Da Prato and Sinestrari [13] and the explicit formula in [25], at least for appropriate initial conditions $\left({ }^{11}\right)$, yielding

$$
u(t)=\varphi(t)+Q(t)(f-\varphi(0))-\int_{0}^{t} Q(t-s) \varphi^{\prime}(s) d s+\int_{0}^{t} Q(t-s) F(s) d s .
$$

Even for general $f, \varphi, F$, the solution (17) gives the unique "optimal regular" generalized solution (see [29]), is always $C^{1}$ on $] 0, \infty[$, and always takes on the initial value $f$ in at least an appropriate generalized sense (see [29], [30]).

In what follows we take for simplicity $F=0$. If $B f \neq \varphi(0)$ we have at least a "shock" at $t=0$. We shall now always write $B f=\varphi\left(0_{-}\right), \varphi(0)=\varphi\left(0_{+}\right), \varphi\left(0_{+}\right)-\varphi\left(0_{-}\right)=c_{0}$. So for $\varphi$ constant, with $c_{0}$ in general $\neq 0$,

$$
u(t)=\varphi+Q(t)(f-\varphi)=\varphi+Q(t)\left(f-\varphi\left(0_{-}\right)\right)-Q(t) c_{0}
$$

is the generalized solution, with the shock represented by $-Q(t) c_{0}$. Periodic shocks, periodic heat shocks, were studied in detail via generalized solutions in [29], [30], and in the latter applications to solar cells in spinning satellites, accelerated testing, were made.

Starting in 1994 there was initiated the study by asymptotic methods of such problems with discontinuous boundary behavior (some preliminary research on this is in [31],

$\left.{ }^{11}\right) C^{1}$ solutions exist on $\left[0, \infty\left[\right.\right.$ iff $f-\varphi(0) \in D(A), A(f-\varphi(0))+F(0)-\varphi^{\prime}(0) \in \overline{D(A)}$, mild solutions iff $B f=\varphi(0) ; F$ and $\varphi$ are assumed to be in $C^{1}$. 
but otherwise, i.e. essentially all, this work is still unpublished) $\left({ }^{12}\right)$. For the just above mentioned shocks situations this consists in: (i) "connecting" in "small" intervals of time $\left[0, \eta[\right.$ (where $\eta$ will tend to 0$) \varphi\left(0_{-}\right)=B f$ (or $B f_{n}$ with $f_{n}$ near $f$ ) to $\varphi$, i.e. replacing the given $t$-dependent discontinuous boundary condition $\left(\varphi\left(0_{-}\right), \varphi(t) t>0\right)$ by the "fast varying" continuous boundary conditions $\varphi_{\eta}(t)=\varphi(t)+\psi_{\eta}(t)$, supp $\psi_{n} \subset[0, \eta[$, approaching in an appropriate sense the discontinuous data, $\varphi_{\eta}$ (i.e. $\psi_{\eta}$ ) being such that for it a classical (or mild) solution exists up to and including $t=0$, (ii) computing the solutions $u_{\eta}$ corresponding to the $\varphi_{\eta}$, and seek the limit (if it exists) as $\eta \rightarrow 0$ of the $u_{\eta}, u$, as being the solution to the problem with the given discontinuous initial value-boundary value data.

To understand better the scope and possibilities of this asymptotic approach (problems that can be treated, future possible developments) let us return, in (i) of the above paragraph, to the matter of " $\varphi_{\eta} \ldots$ approaching in an appropriate sense the discontinuous data". So in the above considered shock situation (13), where the initial-boundary data only contain information expressing a "jump" $c_{0}=\varphi\left(0_{+}\right)-\varphi\left(0_{-}\right)$at $t=0$ (as in the passage, transition, through an interface separating two media) $\left({ }^{13}\right)$, the way the $\varphi_{\eta}$, or equivalently the $\psi_{\eta}$, approach (tend to) the given data as $\eta \rightarrow 0$, must express this. The conditions: $\left\|\varphi_{\eta}\right\| \leq$ a constant and $\psi_{\eta}^{\prime} \rightarrow c_{0} \delta$ in $\mathcal{E}^{\prime}$ will do that in the "jump" situation being considered $\left({ }^{14}\right)$.

If there is interaction at $t=0$ (say for example a "heat explosion" occurs at $t=0$ by brief violent chemical reaction at the interface transition) this must appear in the statement of the problem (and correspondingly the above conditions will be modified so as to express this fact). (13) is then replaced by:

$$
\begin{aligned}
& u^{\prime}=\widehat{A} u+F(t), \\
& u\left(0_{-}\right)=f, \\
& (\sin u)(0)=\sigma, \\
& B u(t)=\varphi(t), \quad t>0,
\end{aligned}
$$

where si stands for "singular interaction" ((13) corresponds to $\sigma=0), \sigma$ is a distribution, or even a hyperfunction (see [20], [34], [16] (analytic functionals)) with support at 0

$\left({ }^{12}\right)$ In the just above described approach, the singular problem (13) is posed and solved in terms of appropriate generalized solutions ( $n$-times integrated solutions). In the asymptotic approach the singular problem $(P)$ is treated as limit of approximating regular problems $\left(P_{\eta}\right)$, $\eta \rightarrow 0$, with "fast varying" boundary conditions producing regular solutions $u_{\eta}$ that tend to the solution $u$ of $(P)$. The solution obtained by either approach, in the situations considered above, is (as it should be) the same. However, the asymptotic approach has recently permitted treatment of problems of a "much higher order of singularity" than those in the situations considered above (where only shocks can occur).

$\left.{ }^{13}\right)$ Essentially a "passive" transition between media, but without sudden addition or subtraction affecting $\varphi$ at $t=0$ (of energy, heat, ...) injected or drained at $t=0$, i.e. a transition without "interaction".

$\left.{ }^{14}\right)$ Though somewhat weaker conditions can also be used, i.e. will lead to the same solution $u=\lim u_{\eta}$. 
( $\sigma \in \mathcal{E}^{\prime}$, or more generally $\sigma \in B_{0}=\{$ hyperfunctions on $\mathbb{R}$ with values in $X$ and support at 0$\}$ ), with now (instead of $\psi_{\eta}^{\prime} \rightarrow c_{0} \delta$ as for (13)) $\psi_{\eta}^{\prime} \rightarrow c_{0} \delta+\sigma^{\prime}$, while $\varphi_{\eta}(0)=B f_{\eta}$, $f_{\eta} \rightarrow f$ in $X,\left(\sigma^{\prime}\right.$ is necessarily of the form $\left.\sum_{k=1}^{\infty} c_{k} \delta^{(k)}\right)\left({ }^{15}\right)$. We have a solution $u$ of (18) if $\psi_{\eta}$ as just described exists such that $u_{\eta} \rightarrow u$.

TheOrem 8. For any $f \in X, \varphi \in C^{1}, F \in L_{\mathrm{loc}}^{1}, \sigma \in B_{0}$, there exists a unique solution $u$ for $(18)$.

The details concerning all these recent results are still unpublished at this time, and further research on this matter is in progress. There are a number of applications to physical and engineering problems with singular interface transitions.

We shall now, as promised towards the end of Section 1, return to matters concerning higher order kernels.

4. $K(t)$-convoluted semigroups and applications. Recently Arendt, El-Mennaoui and Keyantuo [2] studied extensively local $n$-times i.s.g. (loc. $n$-i.s.g.), which correspond essentially to the $\mathcal{S}_{K}(t)$ of Section 1 with $Z_{K}(a)=X(0<a<\infty), K(t)=t^{n} / n$ !, some $n=1,2, \ldots\left({ }^{16}\right)$, and gave generation theorems for such loc. $n$-i.s.g. in terms of the growth of (the norm of) the resolvent $R(z, A)$ in some subdomain of $\{z \in \mathbb{C}: \operatorname{Re} z>0\}$, an "exponential domain" indeed (see [2]) $\left({ }^{17}\right)$. On the other hand, loc. $n$-i.s.g., and the local $n$-times integrated solutions from which they arise, are directly related to distributional solutions (and distribution semigroups, see [22]), a fact which in turn is roughly speaking due to the local structure property of distributions as local derivatives (of some order $n$ ) of $C^{1}$ functions, hence the regularizing role of the " $(n+1)$-integration" convolution kernels $K_{n}(t)=t^{n} / n$ !. Moreover, $K_{n}(t)$ can be identified with the fundamental solution $u$ of

$$
P_{n}(D)=D^{n+1}=d^{n+1} / d t^{n+1} \quad\left(P_{n}(D) u=\delta\right)
$$

having support in $[0, \infty[$.

Now, in more singular problems, where for instance ultradistributions would replace distributions, the local structure properties of the former brings in convolution kernels $K(t)$ of "highter order" instead of the $K_{n}(t), P_{n}(D)=D^{n+1}$ of (19) being then replaced by an ultradifferential operator $P(D), K(t)$ being now identified with the fundamental solution of the latter $P(D)$ (with support in $[0, \infty[$ ), see the recent work of Cioranescu [7], and Cioranescu and the author [8], [9]. So a kernel $K(t)$ associated to a typical ultradifferential operator $P(D)$ (and the corresponding Cauchy problems in terms of ultradistributions of Gevrey class) arises as follows (see [7], [8], [9]): (i) let $M_{k}, k=$ $0,1,2, \ldots$, be a sequence of positive numbers such that $M_{0}=1, M_{k}^{2} \leq M_{k-1} M_{k+1}$ and

$\left({ }^{15}\right)$ As for the precise description of the notion of convergence, topology, in " $\psi_{\eta}^{\prime} \rightarrow c_{0} \delta+\sigma^{\prime}=$ $h \in B_{0}$ ", notice that "moments" are defined for the $\psi_{\eta}^{\prime}$, and $h$ via $m_{k}^{\eta}=\left\langle\psi_{\eta}^{\prime}, z^{k}\right\rangle, m_{k}=\left\langle h, z^{k}\right\rangle$, $k=0,1,2, \ldots$ Then the above convergence is expressed by: $m_{k}^{\eta} \rightarrow m_{k}$ in $\eta$ for each $k=0,1,2, \ldots$, and $\sqrt[k]{\left\|m_{k}^{\eta}\right\|} \rightarrow 0$ in $(\eta, k)$

$\left({ }^{16}\right)$ See [30].

$\left({ }^{17}\right)$ Which may be replaced by a "half-plane" if the local $n$-i.s.g. is indeed a (global) $n$-i.s.g. 
$\sum_{k=1}^{\infty} M_{k-1} / M_{k}<\infty$; (ii) set $m_{k}=M_{k} / M_{k-1}$ for $k \geq 1\left(\right.$ so $\left.\sum_{k=1}^{\infty} 1 / m_{k}<\infty\right)$ and define the entire function

$$
P(z)=\prod_{k=1}^{\infty}\left(1+\frac{z}{m_{k}}\right) .
$$

To the $P(\cdot)$ in $(20)$ corresponds an ultradifferential operator $P(D)$ as mentioned just above, and by inverse Laplace transform of $P(z)$ we obtain the corresponding kernel $K(t)$. This $K(t)$ is a typical example of scalar kernels $K(t)$ "of higher order" among those introduced in Section $1\left({ }^{18}\right)$.

We now describe in the general context of Section 1 but considering only scalar kernels, a very general generation theorem which contains not only the recent one in [2] but earlier results of Beals [3], [4], Chazarain [5], [6], Emamirad [15], Cioranescu and Zsidó [10], Cioranescu [7]; this and other related results are in [8], [9].

Specifically, in the context of section 1 , if $Z_{K}(a)=X$ then $\mathcal{S}_{K}(t)$ (which we write $S_{K}(t)$ in that case) $\in \mathcal{B}(X)$ and we call $\left\{S_{K}(t)\right\}_{0 \leq t<a}$ the $K$-convoluted semigroup ( $K$ c.s.g.) generated by $A$. Even if $A$ does not have the uniqueness property one can define a similar concept of $K$-convoluted semigroup in the extended sense ( $K$-c.s.g.e.) $\left\{S_{K}(t)\right\}_{0 \leq t<a}$ having $A$ as a generator $\left(^{19}\right)$. We assume in what follows $K$ scalar, defined on $\left[0, \infty\left[, K(0)=0\right.\right.$, with $\left|K^{\prime}(t)\right| \leq C e^{\omega t}$ for some $C>0, \omega \geq 0$, and that $\widetilde{K}(z)=\int_{0}^{\infty} e^{-t z} K^{\prime}(t) d t \neq 0$ for $\operatorname{Re} z>0$.

Let $\Phi$ be a real-valued positive function on $\left[r_{0}, \infty\left[, r_{0} \geq 0\right.\right.$, in $C^{1}$ with $\Phi^{\prime}>0$, and $\lim _{r \rightarrow \infty} \Phi(r)=\infty$. Define

$$
\chi(\Phi)=\chi=\varlimsup_{r \rightarrow \infty} \frac{\Phi(r)}{r}, \quad \sigma(\Phi)=\sigma=\varlimsup_{r \rightarrow \infty} \frac{\ln r}{\Phi(r)}, \quad \mu(\Phi)=\mu=\varliminf_{r \rightarrow \infty} \frac{\ln r}{\Phi(r)} .
$$

For $\alpha, \beta>0$, we define the subdomain $\Gamma_{\alpha \beta}(\Phi)$ of $\{\operatorname{Re} z>0\}$ by

$$
\Gamma_{\alpha \beta}(\Phi)=\Gamma_{\alpha \beta}=\{z \in \mathbb{C}: \operatorname{Re} z \geq \beta, \operatorname{Re} z \geq \alpha \Phi(|z|)\}
$$

We have the following generation theorem:

Theorem 9. If for $0<\alpha<\chi^{-1}, \beta, \ell>0,-1<\gamma<\ell-\sigma$, one has $\Gamma_{\alpha \beta} \subset \rho(A)$ and

$$
|\tilde{K}(z)|=O\left(e^{-\ell \Phi(|z|)}\right), \quad\|R(z, A)\|=O\left(e^{\gamma \Phi(|z|)}\right), \forall z \in \Gamma_{\alpha \beta},
$$

then $A$ generates a $K^{[1]}$-c.s.g.e. on $\left[0, a\left[\right.\right.$, with $a=(\ell-\gamma-\sigma) \alpha^{-1}$ and $a K^{[1]}$-c.s.g. if $\chi=0\left({ }^{20}\right),\left({ }^{21}\right)$.

$\left({ }^{18}\right)$ There are examples of still "more general" kernels $K(t)$; all these kernels are connected to interesting applications.

$\left({ }^{19}\right)$ See [9]. We can also say that " $A$ generates $\left\{S_{K}(t)\right\}_{0<t<a}$ ".

$\left({ }^{20}\right) \rho(\cdot)$ denotes as usual the resolvent set; $K^{[1]}(t)=\left(D^{-1} K\right)(t)=\int_{0}^{t} K(s) d s$.

$\left({ }^{21}\right)$ For more details, a result in the opposite direction, examples and applications, see [9]. See also the recent papers by Keyantuo [18], [19], for other examples. 
5. Some research directions and problems. We mention briefly a few (among many) $\left({ }^{22}\right)$ directions in which further research in the areas described in this article is progressing at present, and some of the corresponding open questions.

One direction concerns the matter of investigating completely general single equations of the type (1) above directly or rather in $K(t)$-regularized form (2), or similarly investigating evolution operators $\mathcal{S}_{K}(t)$ on arbitrary subspaces $Z$ of $Z_{K}$ including 1-dimensional spaces $Z$ (i.e. again single equations), in particular from the point of view of generation theorems. Among the people working on this, Frank Neubrander, and the author, have obtained results very recently, following each one somewhat different routes, using local resolvents, and alternatively approximate resolvents on subdomains $\Gamma_{\alpha \beta}(\Phi)$ of $\{\operatorname{Re} z>0\}$; further research is in progress.

Another wide open direction of further work concerns the singular transition problems (s.t.p.) discussed in Section 3 above. Problems in this area concern: the classification of s.t.p. in problems with finite order singularities (f.o.s.) or infinite order singularities, characterization of f.o.s., in particular in an "observable sense", i.e. in terms of the solution $u$ of the given problem; also the investigation of the inverse problem for s.t.p. (feasibility in principle, and possibly explicit formulas); investigation of physical phenomena and engineering mathematical models in which s.t.p. of "higher order" than shocks and/or heat explosions occur (the latter is of course a natural objective and some research on this is under way).

The above are, however, as already said, only some examples of research directions and problems in the areas discussed in the present article.

\section{References}

[1] W. Arendt, Vector-valued Laplace transform and Cauchy problems, Israel J. Math. 59 (1987), 327-352.

[2] W. Arendt, O. El-Mennaoui and V. Keyantuo, Local integrated semigroups: evolution with jumps of regularity, J. Math. Anal. Appl. 186 (1994), 572-595.

[3] R. Beals, On the abstract Cauchy problem, J. Funct. Anal. 10 (1972), 281-299.

[4] -, Semigroups and abstract Gevrey spaces, ibid. 10 (1972), 300-398.

[5] J. Chazarain, Problèmes de Cauchy dans les espaces d'ultra-distributions, C. R. Acad. Sci. Paris Sér. A 226 (1968), 564-566.

[6] - Problèmes de Cauchy abstraits et applications à quelques problèmes mixtes, J. Funct. Anal. 7 (1971), 386-446.

[7] I. Cioranescu, Local convoluted semigroups, to appear.

[8] I. Cioranescu et G. Lumer, Problèmes d'évolution régularisés par un noyau général $K(t)$. Formule de Duhamel, prolongements, théorèmes de génération, C. R. Acad. Sci. Paris Sér. I Math. 319 (1994), 1273-1278.

[9] - , 一, On $K(t)$-convoluted semigroups, in: Recent Developments in Evolution Equations, Pitman Res. Notes in Math. 324, 1995, 86-93.

$\left({ }^{22}\right)$ Simply mentioning here some questions in which the author is particularly interested in at this time. 
[10] I. Cioranescu and L. Zsidó, $\omega$-Ultradistributions and their applications to operator theory, in: Spectral Theory, Banach Center Publ. 8, 1982, PWN, Warszawa, 77-220.

[11] Ph. Clément, H. Heijmans et al., One parameter semigroups, CWI Monographs, North-Holland, Amsterdam, 1987.

[12] G. Da Prato, Semigruppi regolarizzabili, Ricerche Mat. 15 (1966), 223-248.

[13] G. Da Prato and E. Sinestrari, Differential operators with non dense domains, Ann. Scuola Norm. Sup. Pisa 14 (1987), 285-344.

[14] E. B. Davies and M. M. Pang, The Cauchy problem and a generalization of the HilleYosida theorem, Proc. London Math. Soc. (3) 55 (1987), 181-208.

[15] H. Emamirad, Systèmes pseudo différentiels d'évolution bien posés au sens des distributions de Beurling, Boll. Un. Mat. Ital. (6) 1 (1982), 303-322.

[16] L. Hörmander, An Introduction to Complex Analysis in Several Variables, Van Nostrand, Princeton, 1966.

[17] H. Kellermann and M. Hieber, Integrated semigroups, J. Funct. Anal. 84 (1989), 160-180.

[18] V. Keyantuo, The Weierstrass formula and the abstract Cauchy problem, to appear.

[19] - On the boundary value theorem for holomorphic semigroups, preprint Math. Dep. Univ. of Puerto Rico, Rio Piedras.

[20] H. Komatsu, Ultradistributions I, J. Fac. Sci. Univ. Tokyo Sect. IA 20 (1973), 25-105.

[21] R. deLaubenfels, C-semigroups and the Cauchy problem, J. Funct. Anal. 111 (1993), 44-61.

[22] J. L. Lions, Les semi-groupes distributions, Portugal. Math. 19 (1960), 141-164.

[23] G. Lumer, Solutions généralisées et semi-groupes intégrés, C. R. Acad. Sci. Paris Sér. I Math. 310 (1990), 577-582.

[24] - Applications de solutions généralisées et semi-groupes intégrés à des problèmes d'évolution, ibid. 311 (1990), 873-878.

[25] —, Problèmes dissipatifs et analytiques mal posés : solutions et théorie asymptotique, ibid. 312 (1991), 831-836.

[26] —, A (very) direct approach to locally lipschitz integrated semigroups and some new related results oriented towards applications, via generalized solutions, in: LSU Seminar Notes in Functional Analysis and PDES 1990-1991, Louisiana State University, Baton Rouge, 1991, 88-107.

[27] —, Semi-groupes irréguliers et semi-groupes intégrés: application à l'identification de semi-groupes irréguliers analytiques et résultats de génération, C. R. Acad. Sci. Paris Sér. I Math. 314 (1992), 1033-1038.

[28] - , Problèmes d'évolution avec chocs (changements brusques de conditions au bord) et valeurs au bord variables entre chocs consécutifs, ibid. 316 (1993), 41-46.

[29] —, Models for diffusion-type phenomena with abrupt changes in boundary conditions, in Banach space and classical context. Asymptotics under periodic shocks, in: Evolution Equations, Control Theory and Biomathematics, Lecture Notes in Pure Appl. Math. 155, Marcel Dekker, New York, 1994, 337-351.

[30] - Evolution equations: Solutions for irregular evolution problems via generalized solutions and generalized initial values. Applications to periodic shocks models, Annales Saraviensis 5 (1) (1994), 1-102.

[31] - Singular problems, generalized solutions, and stability topologies, in: Partial Differential Equations. Models in Physics and Biology, Math. Res. 82, Akademie Verlag, Berlin, 1994, 204-215. 
[32] I. Miyadera, On the generators of exponentially bounded C-semigroups, Proc. Japan Acad. Ser. A Math. Sci. 62 (1986), 239-242.

[33] F. Neubrander, Integrated semigroups and their applications to the abstract Cauchy problem, Pacific J. Math. 135 (1988), 111-155.

[34] P. Shapira, Théorie des hyperfonctions, Lecture Notes in Math. 126, Springer, 1970.

[35] E. Sinestrari, On the abstract Cauchy problem of parabolic type in spaces of continuous functions, J. Math. Anal. Appl. 107 (1985), 16-66.

[36] E. Sinestrari and W. von Wahl, On the solutions of the first boundary value problem for the linear parabolic equations, Proc. Roy. Soc. Edinburgh Sect. A 108 (1988), 339-355.

[37] H. Thieme, Semiflows generated by Lipschitz perturbations of non-densely defined operators, Differential Integral Equations 3 (1990), 1035-1066.

Added in proof. Concerning what is said, in Section 3, in the sentence preceding $\left({ }^{12}\right)$, and the sentence immediately following Theorem 8 , we point out that meanwhile an announcement of a part of the mentioned results (still without proofs) has appeared: G. Lumer, Transitions singulières..., C. R. Acad. Sci. Paris Sér. I 322 (1996), 735-740; and a detailed and extended version (with the proofs) will appear sometime in 1997: G. Lumer, Singular transitions and interactions..., to appear in: Differential Equations, Asymptotic Analysis, and Mathematical Physics, Math. Res. 100, Akademie-Verlag, Berlin. 